\title{
On Generalizations of the Engset Model
}

\author{
Eric W. M. Wong, Senior Member, IEEE, Andrew Zalesky, and Moshe Zukerman, Fellow, IEEE
}

\begin{abstract}
The Engset model has been extensively studied and widely used for blocking probability evaluation in telecommunications networks. In 1957, J. W. Cohen considered two generalizations of the Engset model:

1) Permitting the distributions of the holding time and interarrival time to differ from source to source;

2) Permitting the idle time distribution to depend on whether or not the previous call was successful.

He derived the call and time congestions for the first generalization, however, he simply posed the second generalization as a problem. In a leading teletraffic text by Syski, the call and time congestions are approximated for the second generalization, though it appears that Syski has overlooked that his results are in fact an approximation. In this paper, we point out Syski's apparent oversight, we improve the accuracy of his approximation, and we provide an efficient algorithm for its numerical computation and prove its convergence.
\end{abstract}

Index Terms- Generalized Engset Formula, teletraffic, blocking probability, performance models, state-dependent arrivals.

\section{INTRODUCTION}

$\mathbf{T}$ HE Engset model [5] is well-known, has made its way to many textbooks (eg. [7]), and has been often used in telephony to evaluate blocking probability when the number of traffic sources competing for a limited communication resource is not too large. Furthermore, recent applications to a bufferless optical network [1], [4], [6], [8], [9] and a mobile network that provides packet radio service (GSM/GPRS) [3] have revamped interest in generalized Engset models.

In this paper, we consider Cohen's second generalization [2] of the Engset model in which the distribution of the time until a source generates a new call differs according to whether or not the previous call was successful. This generalization finds application in situations in which a source cannot generate a new call immediately after the arrival of a blocked call, but rather must remain frozen for a certain period.

Considering a particular source, we let $K_{1}$ be the r.v. (random variable) representing the period between the end of transmission of a successful call and the arrival of the next call (successful or not) and $K_{2}$ the r.v. representing the period between the arrival of a blocked call and the arrival of the

Manuscript received November 16, 2006. The associate editor coordinating the review of this letter and approving it for publication was Prof. Maode Ma. The work described in this paper was supported by a grant from the Research Grants Council of the Hong Kong SAR, China [Project No. CityU 1323/04E] and by the Australian Research Council.

E. W. M. Wong is with the Department of Electronic Engineering, City University of Hong Kong, Hong Kong SAR, China (e-mail: ewong@ee.cityu.edu.hk).

A. Zalesky and M. Zukerman are with the Australian Research Council Special Research Centre for Ultra-Broadband Information Networks (CUBIN), EEE Dept., The University of Melbourne, Victoria 3010, Australia (e-mail: $\{$ alz, mzu $\} @$ ee.unimelb.edu.au)

Digital Object Identifier 10.1109/LCOMM.2007.061876. next call (successful or not). In the Engset model, $K_{1}=K_{2}$. In what we call the generalized Engset model, it is possible that $K_{1} \neq K_{2}$.

Interestingly, Cohen posed the $K_{1} \neq K_{2}$ case long before the advent of optical switching and GSM/GPRS. However, in his derivation of the call and time congestions for his generalized Engset model, he reverted to the simpler $K_{1}=K_{2}$ case, though he permitted $K_{1}$ and $K_{2}$ to vary from source to source. In a leading teletraffic text by Syski [7], it appears that Cohen's results have been misinterpreted as also holding for the general $K_{1} \neq K_{2}$ case. In particular, see pp. 173179 in [7], where Cohen's results are re-derived. Although Cohen's results are not exact for the general $K_{1} \neq K_{2}$ case, they may provide an approximation for the call and time congestions. Therefore, Syski's misinterpretation remains useful as an approximation. Although Syski did not intend to introduce an approximation, we refer to his results as Syski's approximation because they do not yield the exact call and time congestions for the $K_{1} \neq K_{2}$ case. Another approximation for call congestion was provided for the $K_{1} \neq$ $K_{2}$ case in [8].

In this paper, we improve the approximation in [8] and show that our new approximation can be numerically computed with a binary search that is guaranteed to converge. Finally, we numerically verify that our new approximation is more accurate than the ones in [7] and [8].

\section{THE MOdeL}

We are interested in Cohen's second generalization of the Engset model. We consider a loss model comprising $N$ sources offering calls to $K$ channels. We assume both $N$ and $K$ are finite integers and $N>K$, but not $N \gg K$. As $N$ becomes large, our model degenerates to an Erlang system, while if $N \leq K$, the call congestion is zero. Neither of these cases interest us here. We assume the length of a frozen period and the length of a period required to transmit a call (holding time) is independent and exponentially distributed with mean $1 / \mu$.

At any time instant, each of the $N$ sources is either active, frozen or idle. A successful call is transmitted during an active period. A source cannot generate a new call during an active or frozen period. An idle period always follows an active or frozen period. We assume the length of an idle period is independent and exponentially distributed with mean $1 / \lambda$. As soon as an idle period expires, the source generates a new call. The new call is blocked if all $K$ channels are engaged, which results in a frozen period following the idle period. Otherwise, the new call is successful if at least one of the $K$ channels is free, which results in an active period.

In terms of our $K_{1}$ and $K_{2}$ notation, we have $K_{1}$ is independent and exponentially distributed with parameter $\lambda$, 
whereas $K_{2}$ is a two stage hypo-exponential distribution, one stage with parameter $\mu$ and the other $\lambda$.

We are interested in determining the call congestion, defined as the steady-state probability that an arbitrary call arrives to find that all $K$ channels are engaged. For small $N$ and $K$, the call congestion can be computed numerically by determining the steady-state distribution for the Markov process underlying our model. The states-space of this Markov process is $\{(i, j) \mid i=0,1, \ldots, K, j=0,1, \ldots, N-K\}$, where state $(i, j)$ means $i$ of the $K$ channels are engaged and $j$ sources are frozen. Hence, $N-i-j$ sources are idle. The steady-state distribution $\pi_{i, j}$ can be computed numerically by solving the set of local balance equations [1], [9]. Finally, the call congestion is given by $\left(T_{o}-T_{c}\right) / T_{o}$, where $T_{o}=$ $\sum_{i, j}(N-i-j)(\lambda / \mu) \pi_{i, j}$ and $T_{c}=\sum_{i, j} i \pi_{i, j}$.

\section{The Approximations}

All three approximations considered in this section reduce the general $K_{1} \neq K_{2}$ case to the $K_{1}=K_{2}$ case for which time congestion can be computed in $O(K)$ using the conventional Engset formula. In particular, the distinct frozen and idle states inherent to the $K_{1} \neq K_{2}$ case are lumped together into what we call a super-idle state. Therefore, the state-space is reduced to $\{j \mid j=0,1, \ldots, K\}$, where state $j$ means $j$ of the $K$ channels are engaged. Hence, $N-j$ sources are in the super-idle state, meaning they are either genuinely idle or frozen. In this way, the call congestion, $\Pi$, for the $K_{1} \neq K_{2}$ case is approximated using the Engset formula with mean oN time $1 / \mu$ and mean OFF time that is equal to the mean length of a super-idle period. Let $m$ denote the mean length of a super-idle period. According to the Engset formula, we have

$$
\Pi=\operatorname{Eng}(m, 1 / \mu, N, K)=\frac{\left(\begin{array}{c}
N-1 \\
K
\end{array}\right) 1 /(m \mu)^{K}}{\sum_{k=0}^{K}\left(\begin{array}{c}
N-1 \\
k
\end{array}\right) 1 /(m \mu)^{k}} .
$$

The mean length of a super-idle period is computed differently for each approximation.

\section{A. Syski's Approximation}

Syski's approximation arose out of his misinterpretation of Cohen's earlier results. In particular, Syski mistook Cohen's results to hold for the general $K_{1} \neq K_{2}$ case.

Let $\Pi$ denote the call congestion. According to Syski (see pp. 100-103 in [7] or pp. 160-161 in [2]), if a source is super-idle at an arbitrary time instant, the next call (successful or not) arrives in $(t, t+d t)$ after this arbitrary instant w.p. (with probability) $\left(1-F_{K_{1}}(t)\right) / k_{1}$ if the previous call was successful and w.p. $\left(1-F_{K_{2}}(t)\right) / k_{2}$ if the previous call was blocked, where $F_{K_{1}}(t)=\mathbb{P}\left(K_{1} \leq t\right), k_{1}=\mathbb{E}\left(K_{1}\right)=1 / \lambda$ and similarly for $K_{2}$. Therefore, if a source is super-idle at an arbitrary time instant, the next call (successful or not) arrives in $(t, t+d t)$ after this arbitrary instant w.p.

$$
g(t) d t=\left(\frac{(1-\Pi)\left(1-F_{K_{1}}(t)\right)}{k_{1}}+\frac{\Pi\left(1-F_{K_{2}}(t)\right)}{k_{2}}\right) .
$$

Writing $g(t)=(1-G(t)) / m$, where $G(t)$ is the distribution of the length of a super-idle period and $m$ its mean, we see

$$
m=\frac{1}{g(0)}=\frac{k_{1} k_{2}}{k_{2}+\Pi\left(k_{1}-k_{2}\right)} .
$$

Substituting $k_{1}=1 / \lambda$ and $k_{2}=1 / \mu+1 / \lambda$ into (3) gives

$$
\frac{1}{m}=\lambda-\frac{\lambda^{2} \Pi}{(\lambda+\mu)} \text {. }
$$

The Engset formula (with mean on time $1 / \mu$ and mean OFF time $m$ ) together with (4) define a pair of coupled nonlinear equations for which it may be possible to numerically compute a solution using successive substitution.

Equation (2) is certainly true in the sense that $d t / m$ is the probability that a present super-idle period terminates after an arbitrary time. However, Syski incorrectly assumed $d t / m$ is also the probability that a present super-idle period terminates after an arbitrary time given that $j$ channels are engaged. Syski overlooked that $m$ is state-dependent. In particular, if $K$ channels were engaged at the beginning of a super-idle period, we have $m=k_{2}$, whereas if fewer than $K$ channels were engaged, we have $m=k_{1}$. But Syski uses $m$ as given by (4) for both cases in equation (2.57) on p. 176 in [7].

\section{B. Approximation in [8]}

In [8], an alternative to (4) is proposed whereby the mean OFF time used in the Engset formula is computed as the weighted average of a genuine idle period and the duration of a genuine idle period plus a frozen period. In particular,

$$
m=\frac{1-\Pi}{\lambda}+\Pi\left(\frac{1}{\mu}+\frac{1}{\lambda}\right)=\frac{1}{\lambda}+\frac{\Pi}{\mu} .
$$

As with Syski's approximation, the Engset formula (with mean ON time $1 / \mu$ and mean OFF time $m$ ) together with (5) define a pair of coupled nonlinear equations for which it may be possible to numerically compute a solution by iterating such that $1 / m_{n}=\Gamma\left(m_{n-1}\right), m_{0}=1 / \lambda$, where $m_{n}$ is the value of $m$ at the $n$th iteration and $\Gamma(m)=\lambda \mu /(\mu+\lambda \operatorname{Eng}(m, 1 / \mu, N, K))$.

In [8], it is shown that $1 / m=\Gamma(m)$ has a unique solution. However, iterating such that $1 / m_{n}=\Gamma\left(m_{n-1}\right)$ is not guaranteed to converge. We remark that the convergence proof in [8] that claims to guarantee convergence of $1 / m_{n}=$ $\Gamma\left(m_{n-1}\right)$ is incomplete. To complete the convergence proof, we would need to prove that the twice iterated operator $\Gamma^{(2)}(m)=\Gamma(\Gamma(m))=1 / m$ has only one solution, namely the solution it inherits from $1 / m=\Gamma(m)$.

\section{Our New Approximation}

To account for the fact that a super-idle period may span multiple frozen periods, we amend (5) such that

$$
m=\frac{1-\Pi}{\lambda}+\Pi\left(\frac{1}{\mu}+\frac{1}{\lambda}+m\right)
$$

that is, $m=(\mu+\lambda \Pi) /((1-\Pi) \mu \lambda)$. Equation (6) is similar to its counterpart (5) except that $m$ has been added to the second term. This change models the possibility that further frozen periods may follow the initial frozen period, whereas (5) wrongly assumes a blocked call is always followed by a successful call.

In the remaining part of this section, we prove a unique solution exists for the set of coupled equations defined by (1) together with (6). This solution is our new approximation for call congestion. We provide a binary search algorithm to 
TABLE I

Call Congestion, $\mu=1$ And $\epsilon=10^{-6}$

\begin{tabular}{ccccc}
\hline \hline$(\rho, N, K)$ & Syski's & {$[8]$} & New & Exact \\
\hline$(0.4,2,1)$ & 0.1629 & 0.1623 & 0.1615 & 0.1528 \\
$(0.9,2,1)$ & 0.2696 & 0.2656 & 0.2597 & 0.2449 \\
$(0.4,3,1)$ & 0.2065 & 0.2061 & 0.2050 & 0.1989 \\
$(0.9,3,1)$ & 0.3316 & 0.3290 & 0.3214 & 0.3149 \\
$(0.4,5,1)$ & 0.2392 & 0.2390 & 0.2379 & 0.2344 \\
$(0.9,5,1)$ & 0.3776 & 0.3764 & 0.3692 & 0.3681 \\
$(0.4,6,3)$ & 0.0319 & 0.0318 & 0.0318 & 0.0302 \\
$(0.9,6,3)$ & 0.1155 & 0.1135 & 0.1125 & 0.1055 \\
$(0.4,9,3)$ & 0.0486 & 0.0486 & 0.0485 & 0.0469 \\
$(0.9,9,3)$ & 0.1628 & 0.1611 & 0.1593 & 0.1544 \\
$(0.4,15,3)$ & 0.0639 & 0.0639 & 0.0638 & 0.0626 \\
$(0.9,15,3)$ & 0.2029 & 0.2019 & 0.1998 & 0.1975 \\
$(0.4,10,5)$ & 0.0078 & 0.0078 & 0.0078 & 0.0074 \\
$(0.9,10,5)$ & 0.0610 & 0.0600 & 0.0598 & 0.0559 \\
$(0.4,15,5)$ & 0.0146 & 0.0146 & 0.0146 & 0.0141 \\
$(0.9,15,5)$ & 0.0995 & 0.0984 & 0.0978 & 0.0943 \\
\hline \hline
\end{tabular}

numerically compute the unique solution for (1) and (6) and we prove its convergence. We have opted for a binary search algorithm because we cannot guarantee that the successive substitution algorithm proposed in [8] converges to the unique solution of (1) and (6). Finally, in the next section, we numerically validate the accuracy of our new approximation relative to Syski's approximation and the approximation in [8] as well as the exact call congestion.

\section{Existence and Uniqueness of Solution for (1) and (6)}

By setting $x=m$ in (6) and moving the left-hand side of (6) to the right, we define the function

$$
f(x)=\frac{1}{\lambda}+\Pi(x)\left(\frac{1}{\mu}+x\right)-x, \quad x \geq 0,
$$

where we have written $\Pi(x)$ instead of $\Pi$ to emphasize that $\Pi$ is functionally dependent through (1) on the mean OFF time, $x$. Our task is to prove $f(x)=0, x \geq 0$, has a unique solution.

To establish solution existence, we observe that $f(x)$ is continuous and changes sign at least once for $x \geq 0$. This is because $f(0)=1 / \mu+1 / \lambda>0$, while as $x$ gets large, the $-x$ term in $f(x)$ dominates, thus $f(x)<0$. Therefore, a solution exists by the intermediate-value theorem.

To establish solution uniqueness, suppose $f\left(x_{1}\right)=f\left(x_{2}\right)=$ 0 for $x_{2}>x_{1} \geq 0$. The mean-value theorem requires the existence of an $\eta$ satisfying $f\left(x_{2}\right)-f\left(x_{1}\right)=f^{\prime}(\eta)\left(x_{2}-x_{1}\right)$, where $x_{1} \leq \eta \leq x_{2}$. This provides us with a contradiction because simple calculations reveal

$$
f^{\prime}(x)=\Pi(x)-1+\Pi^{\prime}(x)\left(x+\frac{1}{\mu}\right)<0,
$$

where the last inequality follows from the fact that $\Pi^{\prime}(x)<0$ for all $x>0$. Therefore, $x_{1}=x_{2}$.

\section{E. Binary Search Algorithm to Solve $f(x)=0$}

Let $x^{*}$ be the unique solution of $f(x)=0$. We define the monotonically decreasing transformation $\Gamma:[1 / \lambda, \Gamma(1 / \lambda)] \longrightarrow[1 / \lambda, \Gamma(1 / \lambda)]$ such that

$$
\Gamma(x)=\frac{\mu+\lambda \Pi(x)}{(1-\Pi(x)) \mu \lambda} .
$$

We show that the binary search algorithm specified in Algorithm 1 finds the unique solution of $f(x)=0$ for an absolute error criterion of $\epsilon$.

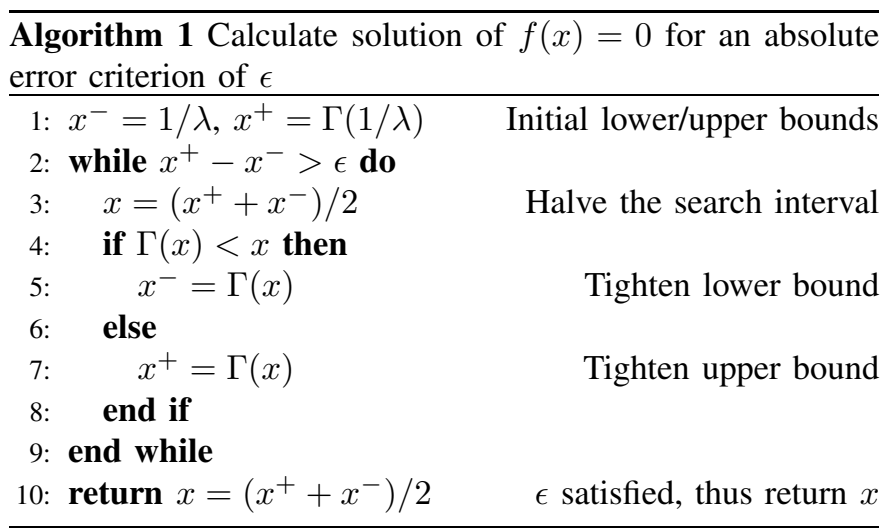

Due to the monotonicity of $\Gamma(x)$, at each iteration of Algorithm 1, if $x<x^{*}$, then $\Gamma(x)>x^{*}$ and thus $\Gamma(x)>$ $x^{*}>x$. Conversely, if $x>x^{*}$, then $\Gamma(x)<x^{*}$ and thus $\Gamma(x)<x^{*}<x$. Consequently, $x^{*}$ lies in the interval $\left[x^{-}, x^{+}\right]$ at each iteration of Algorithm 1. Furthermore, this interval halves at each iteration, thereby ensuring $x^{*}$ is sandwiched within an interval whose eventual length does not exceed $\epsilon$.

\section{NUMERICAL RESULTS}

In Table I, we present plots of call congestion for all three approximations versus what we call the normalized traffic intensity, which is defined as $\rho=(N / K)(\lambda / \mu)$.

Table I empirically verifies that our new approximation is the tightest upper bound on call congestion, while Syski's approximation is the loosest. The approximation in [8] is sandwiched in between.

\section{REFERENCES}

[1] N. Akar and Y. Gunalay, "Stochastic analysis of finite population bufferless multiplexing in optical packet/burst switching systems," IEICE Trans. Commun., vol. E90-B, no. 2, pp. 342-345, 2007.

[2] J. W. Cohen, "The generalized Engset formulae," Philips Telecommun. Rev., vol. 18, pp. 158-170, 1957.

[3] H. Dahmouni, B. Morin, and S. Vaton, "Performance modelling of GSM/GPRS cells with different radio resource allocation strategies," in Proc. IEEE Wireless Communications and Networking Conference 2005, vol. 3, pp. 1317-1322.

[4] A. Detti, et al., "Performance evaluation of a new technique for IP support in a WDM optical network: optical composite burst switching (OCBS)," IEEE J. Lightwave Technol., vol. 20, pp. 154-165, Feb. 2002.

[5] T. Engset, "Die wahrscheinlichkeitsrechnung zur bestimmung der wahleranzahl in automatischen fernsprechamtern" Elektrotechnische Zeitschrift, vol. 39, pp. 304-306, Aug. 1918.

[6] H. Overby, "Performance modelling of optical packet switched networks with the Engset traffic model," Optics Express, vol. 13, pp. 1685-1695, 2005

[7] R. Syski, Introduction to Congestion Theory in Telephone Systems. North Holland, 1959.

[8] H. L. Vu et al., "Scalable performance evaluation of a hybrid optical switch," IEEE J. Lightwave Technol., vol. 23, pp. 2961-2973, Oct. 2005.

[9] M. Zukerman et al., "On teletraffic application to OBS," IEEE Commun. Lett., vol. 8, pp. 116-118, Feb. 2004. 